\section{La producción y su optimice la línea producción de agua}

Production and its optimize water production line
Centro Sur.

Social Science Journal

Julio - Diciembre Vol 4 No 2

http://centrosureditorial.com/ind

ex.php/revista

elSSN: 2600-5743

revistacentrosur@gmail.com

Recepción: 15 julio 2019

Aprobación 19 noviembre 2019

Pag 142 - 148

Atribución/Reconocimiento-

NoComercial-Compartirlgual 4.0

Licencia Pública Internacional -

CC BY-NC-SA 4.0

https://creativecommons.org/lice nses/by-nc-sa/4.0/legalcode.es

\author{
Rogelio Navarrete Gómez ${ }^{1}$ \\ Aksell Joao Ayala Solórzano ${ }^{2}$ \\ Elvis Fabián Mendoza Luna ${ }^{3}$ \\ José Emanuel Narea Farfán ${ }^{4}$
}

\section{Resumen}

El presente proyecto se desarrolla debido a su importancia teórico práctico para la empresa Envasadora de agua LiquiAgua por ser una herramienta que permitirá controlar los costos del proceso productivo y por ende conocer la rentabilidad de la empresa. En la medida en que la competitividad es cada vez mayor, la posibilidad de obtener más utilidades con aumentos de precios es cada vez más difícil, por esto la disminución de los costos se ha convertido en la forma más clara para generar mayores utilidades; por esto se dice que los costos son una buena fuente de ingreso y claro está sin que esta disminución de costos lleve a una disminución de la calidad del producto o servicio. Se tomará en cuenta todas las etapas de la producción desde la obtención de la materia prima hasta llegar al consumir final, para lograr un producto inocuo existen ciertos reglamentos que han sido adoptados en varios países para elaboración de productos. EI MSF (Medidas Sanitarias y Fitosanitarias), OMC (Organización Mundial del comercio), y las normas del Codex Alimentarius confirman las medidas que se debe tomar para elaborar productos inocuos y poder comercializarlos.

\footnotetext{
1 MSc. UTEQ. Quevedo, Ecuador. Orcid: https://orcid.org/0000-0001-7804-401X. Email: rnavarrete@uteq.edu.ec.

2 Estudiante Universitario. UTEQ. Quevedo, Ecuador. Orcid: https://orcid.org/0000-0002-0663-0738. Email: aksellayala11@gmail.com.

3 Estudiante Universitario. UTEQ. Quevedo, Ecuador. Orcid: https://orcid.org/0000-0002-9853-374X. Email: elvis_fabiml@ hotmail.com.

4 Estudiante Universitario. UTEQ. Quevedo, Ecuador Orcid: https://orcid.org/0000-0001-5642-5479. Email: enareaf@gmail.com.
} 
Palabras clave: agua, rentabilidad, empresa

\section{Abstract}

The present project is developed due to its theoretical and practical importance for the LiquiAgua water bottling company, as it is a tool that will allow controlling the costs of the production process and therefore knowing the profitability of the company. As the competitiveness is increasing, the possibility of obtaining more profits with price increases is more and more difficult, for this reason the decrease in costs has become the clearest way to generate greater profits; For this reason, it is said that costs are a good source of income and, of course, this decrease in costs does not lead to a decrease in the quality of the product or service. All stages of production will be taken into account, from obtaining the raw material to reaching final consumption. In order to achieve an innocuous product, there are certain regulations that have been adopted in several countries for product manufacturing. The SPS (Sanitary and Phytosanitary Measures), the WTO (World Trade Organization), and the Codex Alimentarius standards confirm the measures that must be taken to produce safe products and be able to market them.

Key words: water, profitability, company. 


\section{Introducción}

El presente proyecto se lo realizó en una envasadora de agua llamada "LiquiAgua" situada en la avenida Guayaquil, perteneciente al cantón El Empalme, la cual se encarga de purificar, embotellar y vender el líquido vital.

LiquiAgua es una empresa que fue creada el 23 de enero del 2007 por el Sr. Luis Manosalva Venegas quien desarrolló esta propuesta de una implementación de una envasadora de agua para satisfacer las necesidades de los sectores que carecen de agua potable apta para el consumo.

En el transcurso de la elaboración de este proyecto se utilizaron diversas técnicas para obtener información y métodos con los cuales nos permitió deducir los problemas y tomar decisiones que beneficien a la empresa, se manifestó la ausencia de un plan maestro de producción, lo cual impide una satisfactoria planificación y programación en la producción generando problemas como el deterioro del espacio físico interno, control de las demandas en temporadas altas, entre otros. Se utilizaron diversos gráficos, cursogramas y tablas para la representación de los recursos, los procesos, la distribución interna y el plan de producción propuesto.

La implementación del plan de producción propuesto para la empresa lograría resultados favorables como la optimización de los recursos haciendo posible un acrecentamiento de le eficiencia de los procesos de producción de las maquinarias, una eficiente comodidad y desarrollo del trabajo para los empleadores, y lo más importante un control estable de la producción y demandas, y un aumento en las ganancias de la empresa.

La finalidad de este proyecto es elaborar un plan de producción acorde a las necesidades que presente la empresa con el fin de mejorar e incrementar la productividad, eliminar cuellos de botella y establecer una correcta ubicación de las instalaciones existentes.

La mayoría de las empresas envasadoras de agua no cuentan con un plan de producción ya que consideran que es un recurso innecesario, debido a la falta de conocimiento sobre la importancia que este posee sobre las actividades que se realizan. Los problemas casuales relacionados a la producción que pueden ocurrir en una empresa de embasamiento del agua son principalmente potenciar la productividad y mejorar la eficiencia, debido a un crecimiento desmedido de la demanda del producto o factores que influyan en los tiempos de operación de los obreros.

El diagrama de Gantt es una herramienta para planificar y programar tareas a lo largo de un período determinado. Gracias a una fácil y cómoda visualización de las acciones previstas, permite realizar el seguimiento y control del progreso de cada una de las etapas de un proyecto $\mathrm{y}$, además, reproduce gráficamente las tareas, su duración y secuencia, además del calendario general del proyecto. 
Desarrollado por Henry Laurence Gantt a inicios del siglo XX, el diagrama se muestra en un gráfico de barras horizontales ordenadas por actividades a realizar en secuencias de tiempo concretas.

Las acciones entre sí quedan vinculadas por su posición en el cronograma. El inicio de una tarea que depende de la conclusión de una acción previa se verá representado con un enlace del tipo fin-inicio. También se reflejan aquellas cuyo desarrollo transcurre de forma paralela y se puede asignar a cada actividad los recursos que ésta necesita con el fin de controlar los costes y personal requeridos.

\section{Materiales y métodos}

Este tipo de investigación se propone la examinación de la situación actual de la empresa Se realiza un análisis de tipo meticuloso y representativo del área de producción de la empresa envasadora de agua LIQUIAGUA-SA, con el objeto de diseñar e instaurar una propuesta para el progreso y avance de la misma a través del uso eficiente de los recursos con los que cuenta.

Método de observación. - Se lo utilizó a través de una visita técnica realizada en la empresa, con el objetivo de conocer la ubicación de la empresa y la distribución de sus instalaciones, con los datos obtenidos se pudo elaborar un layout en la empresa envasadora de agua LIQUIAGUA-SA.

Método descriptivo. - Se lo aplicó para describir la situación actual de la empresa a través de la elaboración de los diagramas PERT y GANTT con base en los datos obtenidos de parte de la administración de la empresa y de la información obtenida por parte de uno de sus colaboradores se pudo desarrollar el diagrama de hilo.

Método deductivo. - Mediante el método deductivo se identificó la situación actual de la empresa, lo cual se lo aplicó en la elaboración de las conclusiones y recomendaciones a partir de los resultados obtenidos.

Método inductivo. - Este método se lo implementó para la identificación del estado que presentan los recursos de la industria y así postular una solución al problema planteado

Método analítico. - Este método de investigación se lo utilizó para la descomposición del proceso de tratamiento de agua y envasado en bidones, en cada una de sus partes o elementos para observar las causas y los efectos de los problemas que se pueden presentar en el proceso de producción.

\section{Resultados}

Luego de tener una idea clara de los procesos que se realizan en la empresa se elaboró el pronóstico de la demanda para el año 2019, para este pronóstico se pretendía usar el método simulación de Montecarlo pero, debido a que el producto que esta empresa comercializa varía con relación a la temporada de invierno o verano 
de una forma muy drástica y, este método es útil para una demanda de tipo lineal, se recurrió al método de variación estacional con tendencia que es un modelo estadístico que toma en cuenta estas variaciones. Para el cálculo se utilizaron los datos provistos por los directivos de la empresa de la demanda histórica para los años 2016, 2017 y 2018. Luego de aplicarlo se obtuvieron los siguientes resultados de la demanda 2019 para cada mes del año, expresado en la Tabla 1

Tabla 1: Pronóstico de la demanda

\begin{tabular}{lllll}
\hline MES & 2016 & 2017 & 2018 & 2019 \\
\hline Enero & 2400 & 1710 & 2000 & 2246 \\
Febrero & 4650 & 9370 & 9850 & 8827 \\
Marzo & 5350 & 9470 & 9650 & 9102 \\
Abril & 4550 & 9500 & 9750 & 8905 \\
Mayo & 4450 & 8700 & 9500 & 8524 \\
Junio & 6650 & 9780 & 9700 & 9890 \\
Julio & 7700 & 9400 & 9800 & 10240 \\
Agosto & 8900 & 9900 & 9300 & 10758 \\
Septiembre & 3850 & 4550 & 4210 & 4855 \\
Octubre & 3250 & 2600 & 2550 & 3252 \\
Noviembre & 2600 & 1950 & 2900 & 2901 \\
Diciembre & 2450 & 2000 & 3150 & 2976 \\
\hline
\end{tabular}

Con el conocimiento de la demanda esperada se realizó el plan de producción tomando en cuenta la capacidad de producción diaria de la empresa, que es 411 bidones diarios en la demanda alta y 140 en la demanda baja, con lo cual nos queda un inventario final del año de -8000 bidones, es decir no se puede satisfacer la demanda. Por lo cual se presentó una propuesta de solución a este problema aumentando el tiempo de trabajo, en los meses de temporada alta se propone trabajar adicionalmente los días sábados a jornada completa para satisfacer la demanda con lo cual nos queda un inventario final anual de 40 bidones como se muestra en la Tabla 2. 
Tabla 2. Plan de Producción Propuesto

\begin{tabular}{lllll}
\hline MES & DEMANDA & $\begin{array}{l}\text { INV } \\
\text { INICIAL }\end{array}$ & PRODUCCIÓN & $\begin{array}{l}\text { INV } \\
\text { FINAL }\end{array}$ \\
\hline Enero & 2246 & 0 & 3080 & 834 \\
\hline Febrero & 8827 & 834 & 9408 & 1415 \\
\hline Marzo & 9102 & 1415 & 9016 & 1329 \\
\hline Abril & 8905 & 1329 & 9016 & 1440 \\
\hline Mayo & 8524 & 1440 & 10192 & 3108 \\
\hline Junio & 9890 & 3108 & 9800 & 3018 \\
\hline Julio & 10240 & 3018 & 10584 & 3362 \\
\hline Agosto & 10758 & 3362 & 9800 & 2404 \\
\hline Septiembre & 4855 & 2404 & 2940 & 489 \\
\hline Octubre & 3252 & 489 & 3220 & 457 \\
\hline Noviembre & 2901 & 457 & 2660 & 216 \\
\hline Diciembre & 2976 & 216 & 2800 & 40 \\
\hline
\end{tabular}

\section{Conclusiones}

Los diagramas de proceso permitieron analizar y comprender las actividades que se realizan para la producción de los bidones de agua, logrando así tener una descripción precisa de los tiempos, actividades y áreas que componen el proceso para la producción del producto de mayor demanda que se realiza dentro de la empresa, como son los bidones de agua. Mediante la implementación del método de variación estacional con tendencia se desarrolló el pronóstico de la demanda de bidones de agua para el año 2019, el mismo que se realizó con el análisis de la demanda de los tres últimos años que ha presentado este producto. Así mismo, se identificó los meses de mayor demanda, los cuales son febrero, marzo, abril, mayo, junio, julio y agosto. Con los datos obtenidos, se determinó que con la implementación de los días sábados en la jornada de trabajo se aumentará la producción de bidones y por consiguiente se cubrirá la demanda en los meses de mayor requerimiento, generando así mayores ganancias a la empresa. 


\section{Referencias}

Freepik, «Aula Facil,» 0506 2016. [En línea]. Available:

https://www.aulafacil.com/cursos/emprender/guia-facil-para-

emprendedores/el-plan-de-produccion-l26807.

N. Aguirre, «www.obs-edu.com,» 12 Diciembre 2007. [En línea]. Available:

http://www.obs-edu.com/int/blog-project-management/etapas-de-

unproyecto/.

b. Jay Heizer, Dirección de la producción y operaciones, Madrid: PEARSON EDUCACIÓN, S.A, 2008.

Everett, Administración de la poducción y de operaciones, México: PRENDICE HALL HISPANOAMERICANA, 1991

Chiavenato, Introducción a la Teoría General de la Administración, Colombia: McGraw Hill, 1995.

C. VANACLOCHA, Diseño de Industrias Agroalimentarias, Mundiprensa, 2004.

J. R. Matínez, «www.gestiopolis.com,» 10 de octubre 2002. [En línea]. Available: https://www.gestiopolis.com/la-distribucion-en-plan

Liker, J. K. (2006). Las claves del exito de TOYOTA. Barcelona, España: Ediciones 2000.

Hanke, J. E. (2006). Pronosticos en los negocios, octava edicion. Naucalpan de Juarez, Mexico: Pearson Education.

Chapman, S. N. (2006). Planificación y control de la producción. Naucalpan de Juárez, Mexico: PEARSON EDUCACIÓN. 